\title{
On the Nuclear Coupling of Proton and Electron
}

\author{
Volodymyr Krasnoholovets ${ }^{1, *}$, Yuriy Zabulonov ${ }^{2}$, Ihor Zolkin ${ }^{2}$ \\ ${ }^{1}$ Institute of physics, Natl. Acad. Sci., 46 Nauky St., UA-03028 Kyiv, Ukraine \\ ${ }^{2}$ Institute of Environmental Geochemistry under Natl. Acad. Sci. and Ministry for Emergencies and Affairs of Population Protection from \\ the Consequences of Chornobyl Catastrophe, 34-a Acad. Palladin Ave., UA-03680, Kyiv, Ukraine
}

Copyright (C) 2016 by authors, all rights reserved. Authors agree that this article remain permanently open access under the terms of the Creative Commons Attribution License 4.0 International License

\begin{abstract}
We study both experimentally and theoretically the creation of a new physical entity, a particle in which the proton and electron form a stable pair with a tiny size typical for a nucleon. A new theoretical approach to study atomic, sub atomic and nuclear systems is suggested. In the framework of this new approach, which takes into account a submicroscopic concept of physics, we discuss similar experimental results of other researchers dealing with low energy nuclear reactions in a solid, plasma, sonofusion and the electrostatic field generated by piezocrystals. It is shown that the formation of sub atomic particles, which we name subatoms, involves an inerton cloud of an atom from the environment. The inerton cloud, as a carrier of mass, is absorbed by the electron and proton, which strongly couples these two particles in a new stable entity - the subhydrogen. Besides, we have generated a subhelium and argue the existence of subdeuterium. In addition to these subatoms there exist also nuclear pairs formed by a subatom with proton, deuteron and neutron.
\end{abstract}

Keywords Discharge, Plasma, Sonoluminescence, Proton, Deuteron, Electron, Inerton, Neutron, Subatom, Fusion

\section{PACS}

52.20.-j Elementary processes in plasmas

52.55.Pi Fusion products effects (e.g., alpha-particles, etc.), fast particle effects

52.80.Tn Other gas discharges

78.60.Mq Sonoluminescence, triboluminescence

25. Nuclear reactions: specific reactions

25.45.-Z $\quad{ }^{2} \mathrm{H}$-induced reactions

28.20.Fc Neutron absorption

28.20.v Neutron physics

29.25.Dz Neutron sources

28.52.-s Fusion reactors

14.80.-j Other particles (including hypothetical)

13.75.C Nucleon-nucleon interactions (including antinucleons, deuterons, etc.)

\section{Introduction}

The mechanism of sonoluminescence unraveled in Ref. [1] allows us to account for the phenomenon of sonofusion [2-8]. In 2002 and 2004 Taleyarkhan, Nigmatulin and their collaborators presented studies of acoustic cavitation experiments by using a pure deuterated acetone $\left(\mathrm{C}_{3} \mathrm{D}_{6} \mathrm{O}\right)$, deuterated benzene $\left(\mathrm{C}_{6} \mathrm{D}_{6}\right)$, acetone mixtures and some other liquids both deuterated and non-deuterated. The pressure reached 15 bar. In a bubble, which emerged due to the resonance at around $20 \mathrm{kHz}$, they initiated the appearance of nanometre sub-bubbles, or clusters by irradiating the bubble by a flow of neutrons with the energy $14.1 \mathrm{MeV}$. In other experiments they did not use an external source of neutrons but a uranyl nitrate (UN) salt of natural uranium $(99.3 \%$ U-238) dissolved in the liquid studied; nuclear-decay recoiled from dissolved uranyl nitrate. The measured neutron energies from bubble fusion experiments were substantial at $\leq 2.45 \mathrm{MeV}$. In the recent paper [8] the authors also mention a flow of tritium, measured in addition to the observed flow of neutrons. Besides, in all the experiments they measured gamma-radiation emanating from the bubble. All the results were stable near $0^{\circ} \mathrm{C}$ but weakly expressed at a higher temperature.

The researchers [8] explain the observed phenomenon of sonofusion as follows. At the stage of collapse a cluster, which is filled with nanometer bubbles, emits with a frequency of about $2000 \mathrm{~s}^{-1}$ pulses of thermonuclear neutrons with the energy $2.45 \mathrm{MeV}$. The productivity of neutrons is about $10^{5} \mathrm{~s}^{-1}$. In parallel, tritium is also emitted with the same productivity. The authors assume that at the reflection of shock waves from the centers of the bubbles, special spherical zones are formed with a size $\sim 10^{-7} \mathrm{~m}$ in which the temperature reaches $\sim 10^{8} \mathrm{~K}$ and the density is $\sim 10^{4} \mathrm{~kg} \mathrm{~m}^{-3}$. These conditions of high temperature and high density result in acts of thermonuclear fusion.

Nevertheless, in the experiments [2-8] the authors did not at all allude to heating of the liquids studied, which points to a kind of a nonradiative transition in nuclei in the small spherical zones mentioned above. In other words, the 
phenomenon of sonofusion may belong to the class of reactions known as low energy nuclear reactions, or cold fusion.

Literature on low energy nuclear reactions is abundant [9, 10], though there is still no theoretical understanding of the phenomena. Besides, in some low energy nuclear reactions subatomic particles of a novel nature were observed.

Dufour and collaborators [11-14] state the occurrence of the cold fusion reaction by sparking in hydrogen isotopes, where hydrogen triggered exothermal reactions in uranium metal, which resulted in the discovery of a hydrex and deutrex that was considered as a non-proven metastable state of hydrogen and deuterium; the hydrex/deutrex has a nuclear size in the order of femtometers. In the experiments of the Dufour's group no gamma-quanta were measured at the formation of the hydrex/deutrex inside a solid (palladium or other material); the process of their formation was non-radioactive. The Dufour's group examined the excess energy production per atom of hydrogen and values up to 6 $\mathrm{keV}$ were measured. The lifetime of those subatomic particles was estimated as at least several seconds, which is quite enough for the nuclear reaction. Further on Dufour and collaborators [15] introduced a hypole (a coupling of proton and electron with an elliptical orbit) to account for Rossi's cold fusion reaction of $\mathrm{Ni}$ to $\mathrm{Cu}$; they propose a new kind of a pico-chemistry on the basis of a pico-hydride. The pico-hydride is a tiny couple of proton and electron, which is involved in the orbital motion around the nucleus of the atom "A".

Storms [9] comments Dufour and co-workers' results in the following way: Hydrogen or deuterium gas disappeared from the apparatus when a high frequency discharge was generated with palladium used as an electrode; the rate of loss compared to the measured energy is linear and gives approximately $7 \mathrm{keV} /$ atom for $\mathrm{H}_{2}$ and $26 \mathrm{keV} /$ atom for $\mathrm{D}_{2}$. This amount of energy is too large to have a chemical source, albeit still small for nuclear reactions. The lifetime of this new form of hydrogen is not short, it looks as rather stable.

Mills [16-22] and collaborators investigated the formation of plasma in a hydrogen gas under special conditions, a so-called rt-plasma, due to a novel reaction of atomic hydrogen that produced, as chemical intermediates, hydrogen in new states that would be at lower energies than the traditional "ground" $(\boldsymbol{n}=\mathbf{1})$ state. Mills named the phenomenon the "BlackLight Process." In the process, energy is released as the electrons of hydrogen atoms are induced by a catalyst (atoms of gaseous tungsten and strontium, which evaporated in the hydrogen gas by heating of thin metal filaments) to transition to lower-energy levels, i.e. drop to lower base orbits around each atom's nucleus, which by Mills' hypothesis corresponds to fractional quantum numbers. The new state of hydrogen was named a hydrino whose binding energy is much larger than in the conventional ground state of the hydrogen atom. The energy saved in the hydrino is intermediate between chemical and nuclear. No harmful radiation was detected. The BlackLight process, i.e. the formation of hydrinos, occurred in a plasma with a low field, 1 to $2 \mathrm{~V} / \mathrm{cm}$ at low temperatures.

New entities were observed in a plasma arc in a hydrogen gas by Don Borghi [23] and Santilli [24]. The parameters of the electric arc were: $27 \mathrm{~V}$ and $30 \mathrm{~A}$. The observed new entities were called pseudoneutrons, as the gauging equipment perceived them as a flow of neutrons at $10^{4}$ counts per second. Being absorbed, those entities caused nuclear transmutations. Thus Santilli's scrupulous studies confirm Don Borghi's rejected observations of the 1960s, which were published much later [23], as well as other experimental results on a possible fusion of the proton and electron into a neutron [24]. Santilli argues the necessity of the broadening of quantum mechanics to describe the detected new quantum mechanical bound state of protons and electrons at short distance, around $10^{-15} \mathrm{~m}(1 \mathrm{fm})$, and he notes this would be an intermediate step prior to the full neutron synthesis through the involvement of a hypothetic particle, etherino, to change the spin state of the boson pseudoneutron.

Conte and Pieralice [25] also pointed to a possible fusion of the proton and electron into a neutron based on their experimental results.

Storms [9] lists many other theoretical works hypothesizing the formation of a neutral particle in which super-heavy electrons enter an orbit close to the proton. In those models an electron gains an increase in mass, which means it acquires energy. In other words, an ultra-speed electron has to increase in mass, as the Lorentz factor $\boldsymbol{m}=\boldsymbol{m}_{\mathbf{0}} / \sqrt{\boldsymbol{1 - v ^ { 2 } / \boldsymbol { c } ^ { 2 }}}$ prescribes (this is the tessel-lattice pushing back). If the electron's orbit is very close to the proton, the electron will shield the proton's nuclear charge. These models being realistic would solve the challenge of low energy fusion. However, Storms (Ref. 9, 137) notes: "The manner by which this energy is accumulated and stored by the electron is critical to predicting its behavior. Failure to address this problem is the weakest part of the proposed models".

In addition, some other interesting results obtained by researchers working in the area of nuclear transformations in condensed media should here be noted. In particular, Urutskoev et al. $[26,27]$ specify that they fixed a "strange" radiation of unknown nature. Storms (Ref. 9, p. 129) refers to a number of researchers dealing with low energy nuclear reactions who have come to the conclusion that new kinds of particles are possibly created as a result of such reactions. Among twelve major observations needed to be addressed by theory, in particular, he writes (Ref. 9, p. 175): "Strange emissions are occasionally observed having behavior much different from normal particles and radiation".

All the experimental and theoretical results mentioned above, i.e. the results associated with the generation of enigmatic neutrons, gamma-rays, tritium, problematic strong electron-proton coupling and the observance of a strange radiation and energy related to this radiation, are highly intriguing and call for a science behind the conventional paradigm accepted in quantum and particle physics. The 
present work is focused on this challenge.

\section{Our experiment on the generation of mysterious particles}

In a series of experiments we have studied emissions from a hydrogen gas, air and a helium gas, all affected by an electric discharge.

A sophisticated high-voltage set-up has been designed to form discharges in a gaseous atmosphere. The set-up consists of two units: a converter unit, i.e. generator and a power supply. The principle of operation of the set-up is to convert the low voltage signal to a high voltage pulsed signal. After the step-up transformer, further rectification of the high voltage is applied through proprietary rectifier circuits to obtain the high-voltage pulses we require. The master oscillator generates high-voltage antiphase control pulses with a frequency of $1 \mathrm{kHz}$ and "dead" time of about 3 to $6 \%$ of the working cycle. These pulses create plasma in the gap filled with a gas between the electrodes. Regulation of the output voltage is achieved by changing the voltage $V$ of the power supply between 12 to $35 \mathrm{~V}$. The output voltage $V$ induces a pulling electric field in the gap between the electrodes. The current in the plasma can vary from 0.01 to $1.5 \mathrm{~A}$. Typical values of the current used in our experiments did not overcome $0.1 \mathrm{~A}$.

Discharges were carried out in a special hermetically sealed stainless steel reaction chamber with a volume of about $250 \mathrm{ml}$, Fig. 1. The chamber can be filled with different gases. We carried out experiments in the hydrogen atmosphere, at the pressure 0.6 to 1 bar, and in the atmosphere of air, which included a water gas as well. We also conducted experiments in a helium atmosphere. By our estimate, the discharge chamber filled with hydrogen at normal conditions contained up to 100 times more hydrogen atoms than is the case with air.

For the measurement of gamma-radiation we used a gamma-ray spectrometer 'Vector' designed by our team, which was adjusted to measure an integral activity of gamma radiation in a range up to $3 \mathrm{MeV}$.

For the measurement of a possible flow of neutrons we used a unit for detection of neutron radiation BDPN-07 (produced by Zakhidprylad Ltd., Lviv, Ukraine). The measurement range of thermal neutron flux density is 10 neutrons $/\left(\mathrm{cm}^{2} \cdot \mathrm{min}\right)$ to $10^{5}$ neutrons $/\left(\mathrm{cm}^{2} \cdot \mathrm{min}\right)$. The measurement range of fast neutron flux density is 50 neutrons $/\left(\mathrm{cm}^{2} \cdot \mathrm{min}\right)$ to $10^{5}$ neutrons $/\left(\mathrm{cm}^{2} \cdot \mathrm{min}\right)$. The energy range of registered neutrons is from $0.025 \mathrm{eV}$ to $14 \cdot 10^{6} \mathrm{eV}$. Sensitivity of the unit BDPN-07 to detect neutron radiation: 4.5 (pulses $\left.\cdot \mathrm{cm}^{2}\right) /($ neutron) for fast neutrons and 13.0 (pulses $\left.\mathrm{cm}^{2}\right) /($ neutron) for heat neutrons. The maximum exposure dose of gamma radiation, which does not introduce additional error in measuring the neutron flux density, is 10.0 $\mathrm{mR} /$ hour. The probability of the density of neutron flux measured by the device is at the level of 0.95 .

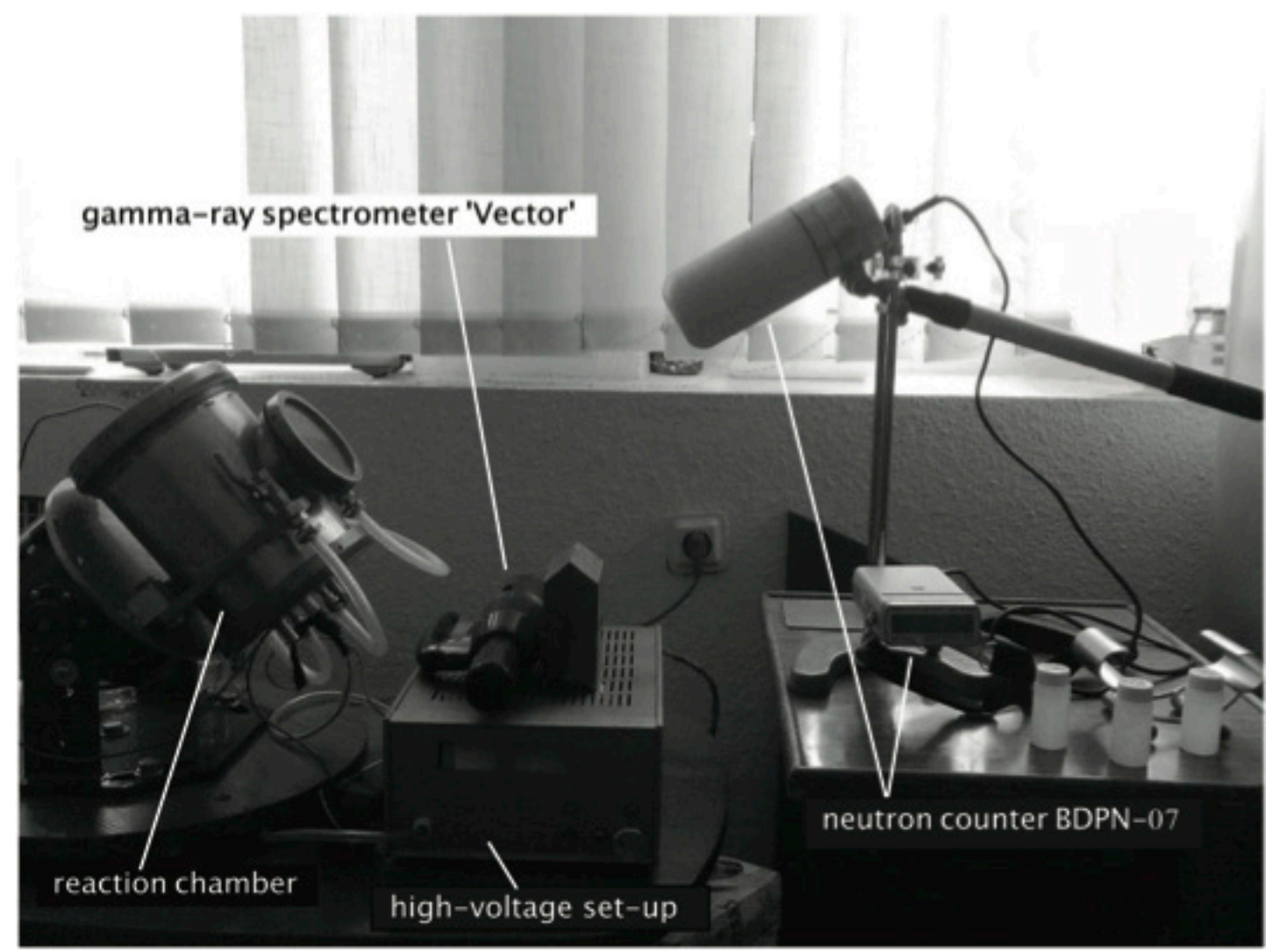

Figure 1. Experimental set-up for studying the emission of exotic particles. 
We also used a passive-type track-edge fast neutron plastic detector CR-39.

In addition we used a device 'Rudra' for measuring inertons, which measures mass flux (Fig. 2), and which was designed by our team as a scintillator of inerton signals absorbed by the antenna. 'Rudra' is able to operate also as a spectrometer measuring frequencies of inertons in a range from a few $\mathrm{Hz}$ to $100 \mathrm{kHz}$. In the experiments, the device was adjusted to measure the integral activity of inertons.

In the reaction chamber, needle electrodes were made of tungsten. The gap between the electrodes varied from $1 \mathrm{~mm}$ to $40 \mathrm{~mm}$.

We observed that the discharge in the hydrogen gas and the air produced a bright blue light, though in the case of the helium gas the light was purple (Fig. 3). The light intensity did not change with the change of the voltage level, which we applied to the electrodes.

After launching a discharge in the reactor chamber filled with the gas studied, we manipulated the tuning knob, gradually increasing the output voltage $V$ from 12 to $35 \mathrm{~V}$. Between 16.5 and $17.5 \mathrm{~V}$ the three devices arranged near the reaction chamber measured signals. When the voltage level became higher than $17.5 \mathrm{~V}$ and up to the maximum value of $35 \mathrm{~V}$, no signals were measured by the gamma-ray measuring device 'Vector' and the neutron-measuring unit BDPN-07. The inerton measuring device 'Rudra' recorded signals over the entire range of voltage changes.

The emission of three kinds of different particles was observed only in the case of plasma produced by a DC pulsed voltage. When the high-voltage set-up was adjusted producing plasma by using an AC voltage, no emission of gamma quanta and neutrons was measured.

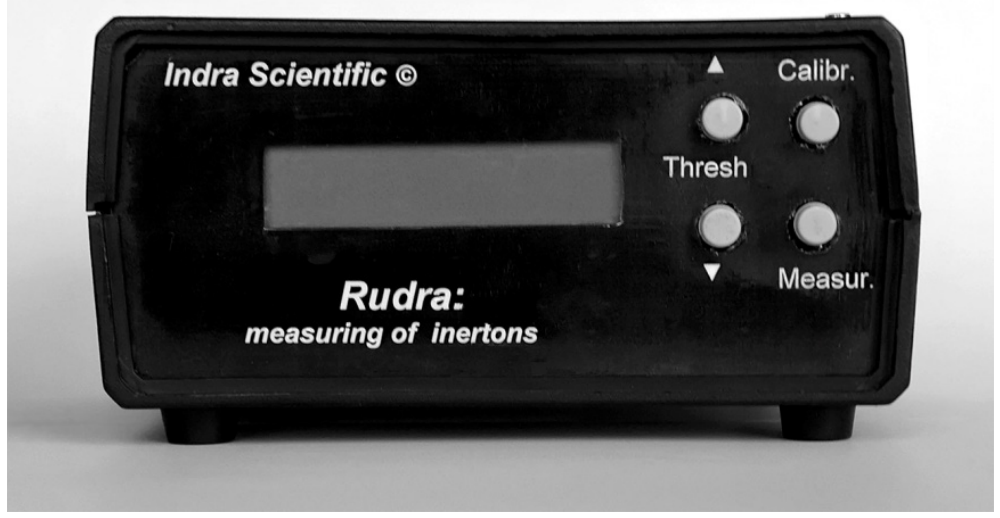

Figure 2. Inerton measuring device 'Rudra'.

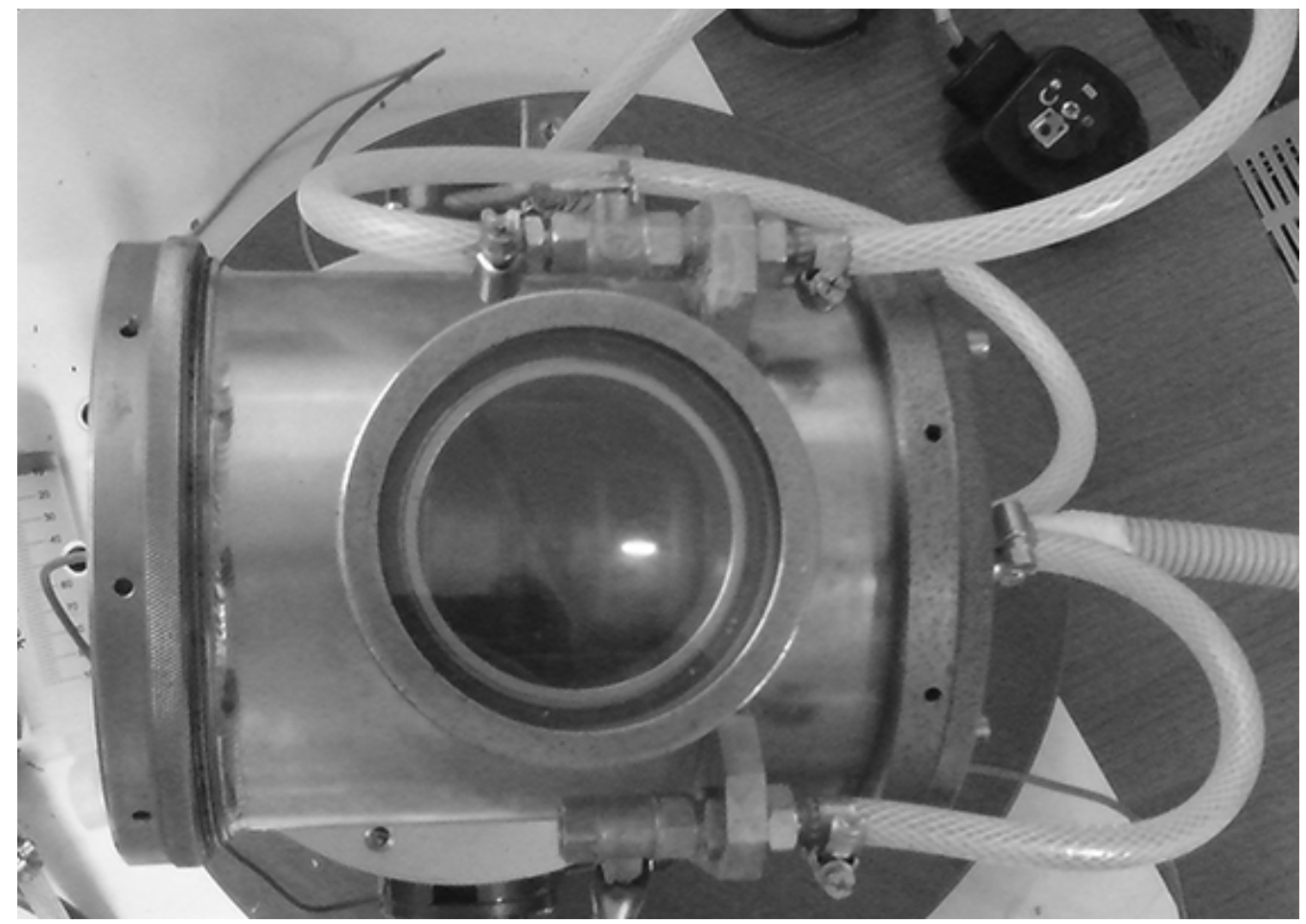

Figure 3. Discharge in the reaction chamber filled with a helium gas. The gap between the tungsten needle electrodes is $8 \mathrm{~mm}$. 

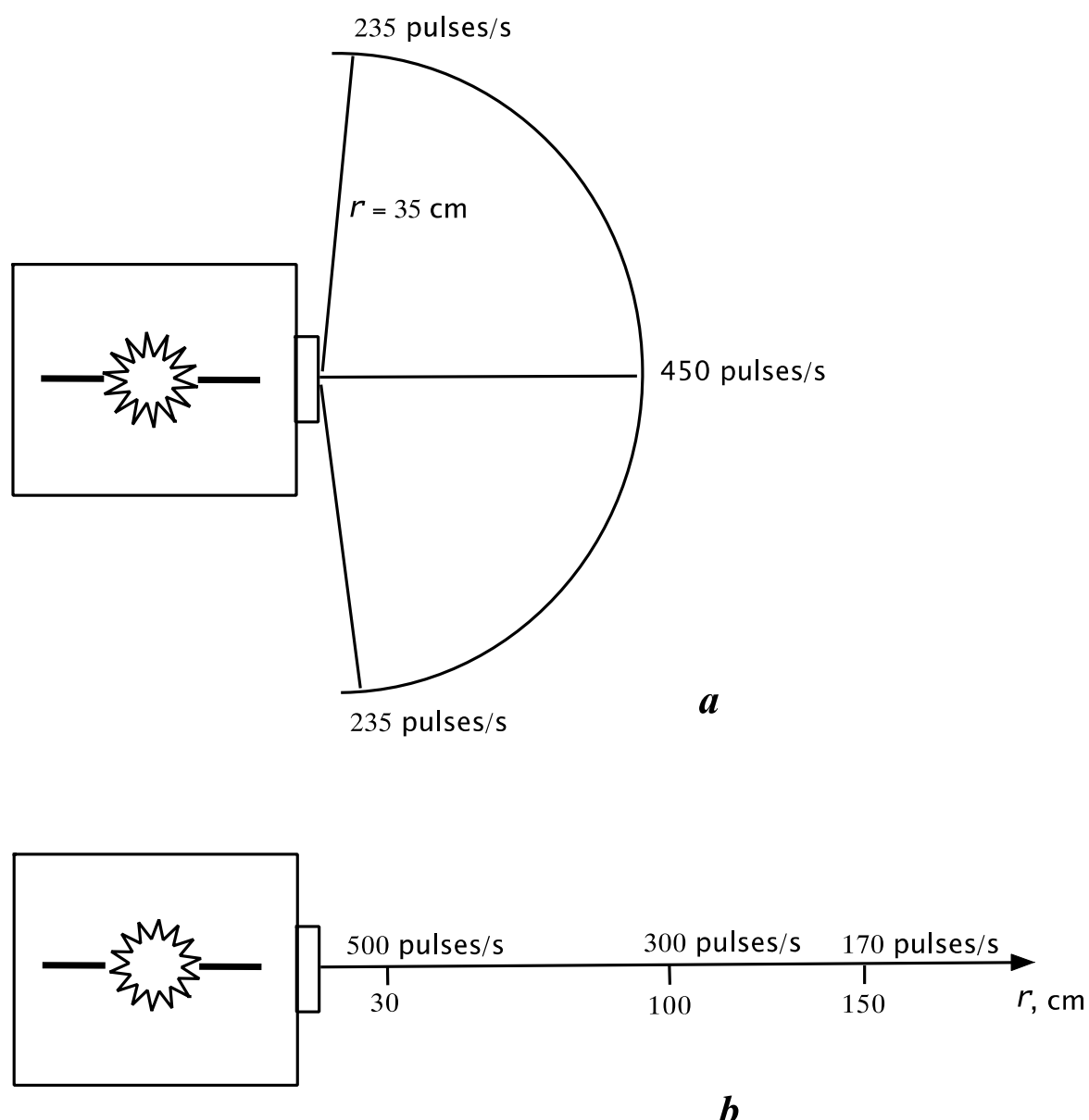

Figure 4. Gamma-radiation measured at the resonance discharges in the reaction chamber, which is emitted through the window. The gap between the electrodes is $6 \mathrm{~mm}$.

Table 1. Measurement of neutron flux density.

\begin{tabular}{|c|c|c|}
\hline The gap between the electrodes, $\mathrm{mm}$ & $\begin{array}{l}\text { Neutron radiation near the windows } \\
\text { of the reaction chamber, } \\
\text { neutrons } /\left(\mathrm{cm}^{2} \cdot \mathrm{min}\right)\end{array}$ & $\begin{array}{l}\text { Neutron radiation at 1 m from the } \\
\text { reaction chamber window, } \\
\text { neutrons } /\left(\mathrm{cm}^{2} \cdot \mathrm{min}\right)\end{array}$ \\
\hline 4 & $(2.5-3) \times 10^{5}$ & $7 \times 10^{3}$ \\
\hline 6 & $(2.5-3) \times 10^{4}$ & $7 \times 10^{3}$ \\
\hline 8 & $5 \times 10^{3}$ & $8 \times 10^{2}$ \\
\hline 11 & 8 to 10 & 8 to 10 \\
\hline
\end{tabular}

Figure 4 shows the distribution of the density of gamma-radiation emanating from the window of the reaction chamber at different distances and different angles. The excess of gamma-ray radiation above the background is quite noticeable, namely, more than three times in the vicinity of the window.

The neutron flux density was measured in the vicinity of the window of the reaction chamber and at a distance of $1 \mathrm{~m}$ from the window. The results obtained at the measurement of discharge in the hydrogen atmosphere are demonstrated in Table 1 (almost the same results were obtained in the air atmosphere too). The background neutron radiation varied from 8 to 10 neutrons $/\left(\mathrm{cm}^{2} \cdot \mathrm{min}\right)$.

So at a strong discharge the neutron counter measured $10^{3}$ pulses per second at a distance of $1 \mathrm{~m}$ from the window of the reactor chamber. At a distance of about $15 \mathrm{~cm}$ from the window the device measured the maximum value that it could measure, $3 \times 10^{5}$ neutrons $/\left(\mathrm{cm}^{2} \cdot \mathrm{min}\right)$. When the generator was switched off or was turned to a higher or lower voltage level outside the resonance range of 16.5 to $17.5 \mathrm{~V}$, the neutron counter measured only the background neutron radiation.

Very similar results were obtained when we examined a helium gas.

No tracks were observed in the six used fast neutron plastic detectors CR-39. This points out to the fact that the reaction chamber generated only slow neutrons that the BDPN07 measured. 


\section{Discussion}

\subsection{A new physical field}

Inerton radiation is a new kind of radiation that was introduced in the works of one of the authors (see, e.g. Ref. [28]), and which is associated with the field of inertia of particles and macroscopic objects. The origin of the inerton field is the interaction of a moving particle with space. As was shown in papers [29-32] the real physical space is a substrate arranged as a mathematical lattice of tightly composed topological balls, which was called the tessellattice. So, a particle moving through the tessellattice becomes surrounded with excitations named inertons [33-35]. The particle's cloud of inertons is mapped to conventional quantum mechanics as the particle's wave $\psi$ function. Inerton field effects manifest themselves in a number of physical systems, which has been proved experimentally [36-39], and are also shown to be responsible for the diffraction of light [40].

Once again, Urutskoev et al. [26, 27] emphasized that they fixed a "strange" radiation of unknown nature. The same mentioned Storm [9].

The discovery of inertons means that they are able to induce the variation of mass of entities and the migration of mass in condensed media [41]. Note some other researchers also raise the issue of the necessity of variable masses in quantum systems and particularly at low energy nuclear reactions; see, e.g. recent works of Davidson $[42,43]$.

In fast processes, such as collisions of particles, radioactive and turbulent acts, some inertons may be released from the particles' and/or atoms' inerton clouds. In such a case free inertons travel in space hopping from lattice cell to lattice cell and can be directly measured, which was demonstrated in work [28].

An inerton is an excitation of space, which is associated with a fractal volumetric constriction of the volume of a cell of the tessellattice; a free inerton transfers mass and fractal properties and is a carrier of the quantum-mechanical interaction, the field of inertia and the field of gravitation. A photon is associated with the surface polarization of a cell of the tessellattice. These two kinds of excitations are basic excitations of the tessellattice, i.e. our universe. The electron's inerton cloud is electrically polarized, as the electron is a charged particle. Hence in the electron's cloud all the inertons are electrically polarized, such that it becomes realistically an inerton-photon cloud [44]. It is this property of the electron's cloud, which allowed one to account for the phenomenon of sonoluminescence [1].

As for the inerton measurement, we can mention that the initial background inerton radiation usually varied from 30 to 40 pulses per second. When the plasma was generated in the reaction chamber, the inerton radiation increased 3 times at a distance of $10-20 \mathrm{~cm}$ from the reaction chamber and gradually dropped to the background value at a distance of about $1.5 \mathrm{~m}$ from the reaction chamber. The distribution of inerton radiation was practically homogenous around the chamber, which means that inertons travelled through metal walls of the chamber as easily as through the chamber's window. We have not seen changes in the measurement of the intensity of inerton flux when the range of voltage changed from 12 to $35 \mathrm{~V}$. The origin of inertons is through any kind of collision of particles in the chamber (for instance, at discharges) when some inertons were stripped from inerton clouds of interacting particles. In terms of the quantum mechanical formalism, this would mean that the particle's wave function was partially split and a certain percentage of it was taken away from the particle.

\subsection{Gamma-radiation}

Thus the neutron detector has measured a mysterious particle. Besides, we have revealed gamma-radiation in the process, which from the viewpoint of usual nuclear physics is impossible in principle. So what is the physics behind the observed phenomena?

Let us start from the consideration of gamma-radiation. What is the origin of gamma-quanta? The previous research [1] on sonoluminescence allows us to understand the mechanism of the gamma-quant generation in detail. Namely, at the collision of a proton with a tungsten atom located on the edge of the cathode, part of the proton's inerton cloud can be shaken off from the proton, such that the proton becomes immobile for a short time until the environment will bring its inertons to the proton, renovating its mobility. In the meanwhile, an electron from the outer shell of the tungsten atom immediately seizes the inertons lost by the proton.

Thus a moving proton consists of two sub systems: the proton core and the proton's inerton cloud. The mass of the whole proton's inerton cloud equals the proton mass $m_{\mathrm{p}}=1.6726218 \times 10^{-27} \mathrm{~kg}$. Due to an inelastic scattering of incoming protons by tungsten atoms of the cathode, a tungsten atom may absorb the whole proton's inerton cloud. More precisely, electrons of the tungsten atom absorb the incoming inerton cloud. Let us use the Bohr model of hydrogen-like nuclei with atomic charge $Z$ to describe the problem of absorption. An average orbit radius of the electron density in the atom might be written as the normalized Bohr radius $r_{\mathrm{B}} / Z$, namely

$$
r_{Z}=\frac{4 \pi \varepsilon_{0} \hbar^{2} \bar{n}^{2}}{e^{2} Z m_{\mathrm{e}}}
$$

where for a tungsten atom $Z=74 ; m_{\mathrm{e}}$ is the electron rest mass; $e$ is the elementary electric charge and $\bar{n}$ is the average orbit number. Let us put $r_{Z}=\mathbf{1 0}^{-\mathbf{1 0}} \mathrm{m}$.

The tungsten atom's electrons absorbing the whole proton's inerton cloud increase their mass to the value of $Z \boldsymbol{m}_{\mathrm{e}}+\boldsymbol{m}_{\mathrm{p}}$. This instantly transfers the electrons to a deeper orbit with an average radius

$$
r_{Z}^{*}=\frac{Z m_{\mathrm{e}}}{\left(Z m_{\mathrm{e}}+m_{\mathrm{p}}\right)} r_{Z} \approx 2.58 \times 10^{-13} \mathrm{~m} .
$$


The disturbed orbit (2) cannot be short-lived, as the big absorbed mass $m_{\mathrm{p}}$ squeezes the electron shells and induces an additional elastic interaction $m_{\mathrm{p}} r_{Z}^{* 2} \Omega^{2} / 2$ in the medium of the electrons' inerton clouds, which stabilize the disturbance. Mass fluctuations in the crystal lattice are rather small [41] and cannot quickly remove the introduced mass defect.

$$
e V=\frac{1}{2} m_{\mathrm{p}} r_{Z}^{* 2} \Omega^{2}
$$

The relationship allows us to estimate the frequency of induced inerton oscillations in the electron density of the tungsten atom: $\Omega \approx 1.5 \times 10^{17} \mathrm{~s}^{-1}$. Since our high-voltage set-up operated in a pulsing mode, every $10^{-3}$ second discharges brought new inerton clouds of the incoming protons to the disturbed atom. The mass of the disturbance gradually increased by portions, $m_{\mathrm{p}}+m_{\mathrm{p}}+m_{\mathrm{p}}+\ldots$ At a threshold number $\boldsymbol{\aleph}$ of incoming protons, which passed their inerton clouds on to the tungsten atom under discussion, the mass becomes $\mathbf{\kappa} \boldsymbol{m}_{\mathbf{p}}$. Since the huge mass is concentrated in electrons of the tungsten atom, which strongly squeezes the electron system to the nucleon, this disturbance should excite the nucleus (Fig. 5). But the excitation of the nucleus means the radiation of a gamma-quantum from it. Hence the kinetic energy of the oscillating mass disturbance is converted to the energy of the nucleus' gamma-quantum
$10^{17}-10^{18}$ protons fall to the cathode per second at the current $0.01-0.1 \mathrm{~A}$. The area of the surface of the cathode edge can be estimated as $\sim 10^{-8} \mathrm{~m}^{2}$. The number of tungsten atoms in this area is around $10^{11}$. Therefore, $10^{6}-10^{7}$ protons fall to each atom of tungsten per second. A part $\aleph$ of these protons may inelastically scatter by one tungsten atom. Suppose $\boldsymbol{N} \approx 10^{4}$. An average velocity typical for electrons of outer shells of an atom may be assumed at $10^{5} \mathrm{~m} \cdot \mathrm{s}^{-1}$. Substituting these values into expression (4) we do get the energy of gamma quantum, $h v_{\gamma \text { quant }} \sim 1 \mathrm{MeV}$.

Once again, we observed no radiation when the high-energy set-up was generating discharges in the reaction chamber in an AC mode, i.e. when the polarity on the electrodes changed with the frequency $10^{3} \mathrm{~Hz}$. This can be accounted for only as follows. The mass disturbance being created after the first collision of the atom with an incoming proton (when the tungsten atom was an absorbing center) was immediately removed at the repolarization of electrodes, as in the opposite phase the tungsten atom became the emitting center.

Santilli [24] did not report on the measurement of gamma-ray radiation in his experiment. This can be explained by the method he used - an electric arc, which establishes a stationary process of proton-electron current. No modulation and pumping of protons, as described above for our case, were applied to the arc; therefore the conditions of the experiment were different from ours.

$$
\frac{1}{2} \aleph m_{\mathrm{p}} v^{2}=h v_{\gamma-\text { quant }}
$$

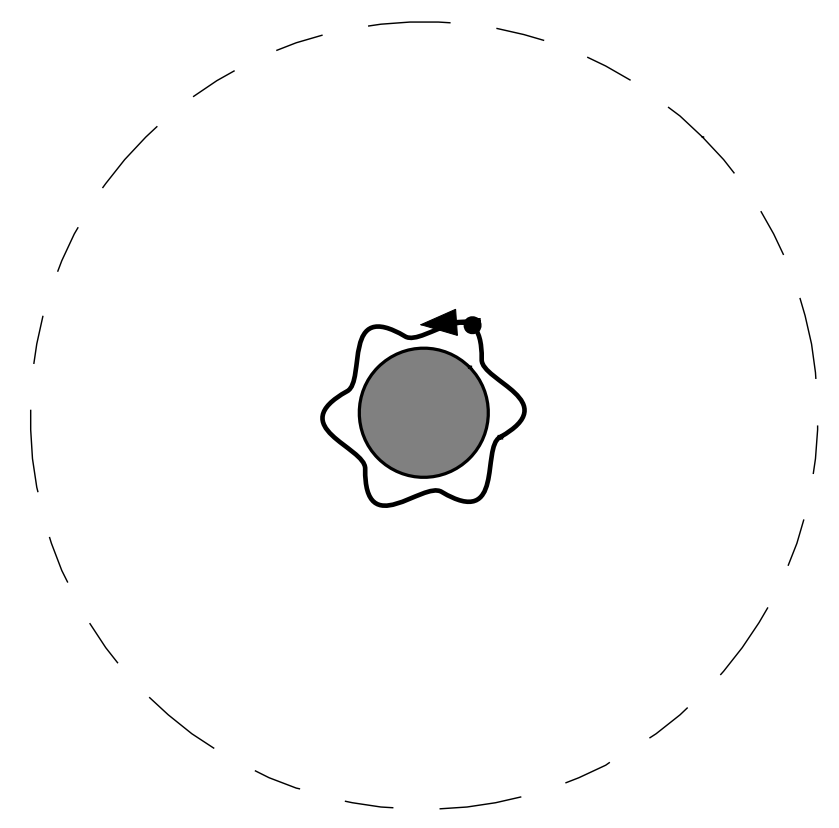

Figure 5. Squeezed disturbed electrons practically touch the nucleon. The dotted line shows the initial unperturbed average orbit. 
On the other hand Taleyarkhan et al. [3] and Nigmatulin et al. [8] reported on gamma-ray emission from collapsed bubbles. In their experiments fast neutrons nucleated 30 bubble clusters per second in deuterated acetone; in self-nucleation experiments in which solute uranium salt emanated $\boldsymbol{\alpha}$ particles, the rate of creation of bubble clusters was much lower (for deuterated benzene-based mixtures and deuterated water). The emergence of sub-bubbles under the affection of a neutron flux (or $\boldsymbol{\alpha}$ particle diffusion flux) reminds of the Rayleigh-Bénard convection in liquids, which occurs in a heated fluid, which leads to the induction of a regular pattern of convection cells known as Bénard cells. Besides, neutrons and $\boldsymbol{\alpha}$ particles should produce defects in the liquids studied, namely, they have to release free deuterons from deuterated molecules of the liquids studied; free deuterons could be assembled in gas bubbles.

The work produced by ultrasound to return the locally compressed liquid to the normal state, which results in collapsing the bubble, can be estimated as [1]

$$
W=V_{2} p_{2}-V_{1} p_{1} ;
$$

here the initial volume is $V_{1}=\mathbf{4} \pi R_{1}^{3} / 3, R_{1}=\mathbf{4 0} \times 10^{-6} \mathrm{~m}$, and the final volume of the collapsed bubble is $V_{2}=\mathbf{4} \pi R_{2}^{3} / 3, \quad R_{2}=10^{-8} \mathrm{~m}$ [8]; the pressures are $p_{1}=p+2 \sigma / R_{1}$ and $p_{2}=p+2 \sigma / R_{2}$ where $\sigma$ is the coefficient of surface tension of acetone, which near $0^{\circ} \mathrm{C}$ might be evaluated as $\sigma \cong 30 \times 10^{-3} \mathrm{~N} \cdot \mathrm{m}^{-1}$. Calculating the work (5), we obtain

$$
W \cong 4 \pi R_{1}^{2} 2 \sigma \cong 1.2 \times 10^{-10} \mathrm{~J} .
$$

In sonofusion the collapse of the bubble occurs at a rate that exceeds the speed of sound at least 10 times, which is a few times faster than is the case of conventional sonoluminescence. Therefore we may anticipate that the released energy is applied to the liquid-bubble boundary layer of the collapsed bubble whose surface area is $4 \pi R_{2}^{2}$. The number of molecules in the boundary layer on the liquid side can be estimated as follows

$$
N=4 \pi R_{2}^{2} / d^{2}
$$

where $d$ may lie between the size average distance of molecules in a liquid and gas. If we put $d=20 \times 10^{-10} \mathrm{~m}$ we get from expression (7): $N \approx 3 \times 10^{2}$.

A sharp impact of the expanding liquid, which has been compressed by ultrasound, falls on these $N$ acetone/benzene molecules. These molecules play the role of a mediator that transfers the released energy (6) inside the bubble filled with gas: due to the very sudden impact, the $N$ molecules shake off their inerton clouds and these $N$ inerton clouds, each having the energy are rushing forward to the gaseous entities of the bubble.

$$
\varepsilon=W / N=4 \times 10^{-13} \mathrm{~J},
$$

The volume of collapsed bubble $V_{2}=4 \pi R_{2}^{3} / 3$ where $R_{2}=10^{-8}$ may include around 500 entities (a mix of acetone and/or benzene molecules and deuteron atoms or deuterons). Some of these entities absorb heavy inertons, as has been discussed above. However, in the case of ultrasound a molecule of gas absorbs the inerton cloud at once, i.e. electrons that wrap the molecules absorb the inerton cloud as a whole (though in the case of discharges in cold plasma the inerton disturbance is accumulated in an atom of the cathode lattice sequentially). The disturbed inerton cloud of the gas molecule excites the nucleon (Fig. 5) and the latter emits a gamma-quantum with the energy of the absorbed inerton cloud, $h v_{\gamma \text { quant }}=\varepsilon=4 \times 10^{-13} \mathrm{~J}$, which corresponds to 2.5 $\mathrm{MeV}$.

\subsection{Neutron radiation}

Having data of Santilli [24] on his arc plasma, we are able to estimate the energy and the momentum of protons that fell on the cathode:

$$
\begin{array}{r}
E=e V=4.38 \times 10^{-18} \mathrm{~J}, \\
m_{\mathrm{p}} v_{\mathrm{p}}=1.2 \times 10^{-22} \mathrm{~kg} \cdot \mathrm{m} \cdot \mathrm{s}^{-1}(9)
\end{array}
$$

where the potential difference was $V=27 \mathrm{~V}$ and hence the velocity of protons $v_{\mathrm{p}}=\sqrt{2 e V / m_{\mathrm{p}}}=7.19 \times 10^{4} \mathrm{~m} \cdot \mathrm{s}^{-1}$. As the cathode and anode were made of tungsten, the kinetic energy and the momentum of a tungsten atom can be estimated as follows

$$
\begin{gathered}
E_{\mathrm{w}}=\frac{1}{2} m_{\mathrm{w}} v_{\mathrm{w}}^{2} \cong 2.07 \times 10^{-20} \mathrm{~J}, \\
m_{\mathrm{w}} v_{\mathrm{w}} \cong 1.2 \times 10^{-22} \mathrm{~kg} \cdot \mathrm{m} \cdot \mathrm{s}^{-1}(10)
\end{gathered}
$$

where the mass of a tungsten atom is $m_{\mathrm{w}}=3.05 \times 10^{-25} \mathrm{~kg}$; the thermal velocity of a tungsten atom $v_{\mathrm{w}}=\sqrt{3 k_{\mathrm{B}} T / m_{\mathrm{w}}} \cong 3.95857 \times 10^{2} \quad \mathrm{~m} \cdot \mathrm{s}^{-1} \quad$ (here the temperature of heated electrodes $T=1150 \mathrm{~K}$ is a fitting parameter).

Comparing expressions (9) and (10) we can observe the momentum resonance between momentums $\boldsymbol{m}_{\mathbf{p}} \boldsymbol{v}_{\mathbf{p}}$ and $m_{\mathrm{w}} v_{\mathrm{w}}$. When the proton impacts the tungsten atom being in antiphase oscillating in its site of the crystal lattice, i.e. when $\boldsymbol{m}_{\mathrm{p}} \vec{v}_{\mathrm{p}}+\boldsymbol{m}_{\mathrm{w}} \vec{v}_{\mathrm{w}}=\mathbf{0}$, both particles must stop. At such conditions, the tungsten atom's inerton cloud is stripped from the atom and the latter becomes momentarily immobile until surrounding atoms bring their inertons to the tungsten atom under discussion and restore its mobility. A free electron, as the lightest particle in the tungsten-gas interface, captures the tungsten atom's released inerton cloud. This is because the effective cross section of the electron, i.e. the size of the cross section of the electron's inerton cloud $\sim \lambda_{\mathrm{dB}} \Lambda \approx \lambda_{\mathrm{dB}}^{2} c / v_{\mathrm{e}}$, much exceeds that of a proton. Thus the electron absorbing the tungsten atom's inerton cloud becomes heavy. Then merging the proton with this heavy electron will result in the creation of a super heavy hydrogen atom. In this system the reduced mass of the proton and the 
electron is almost equal to $\boldsymbol{m}_{\mathrm{p}}$ (in fact $\left.1 / m_{\mathrm{p}}+1 /\left(m_{\mathrm{e}}+m_{\mathrm{w}}\right) \cong 1 / m_{\mathrm{p}}\right)$. Therefore the proton starts to rotate around the heavy electron; the Bohr radius for the rotating proton is

$$
r_{\mathrm{pe}}=\frac{4 \pi \varepsilon_{0} \hbar^{2} n^{2}}{e^{2} m_{\mathrm{p}}}=2.88 \times 10^{-14} \mathrm{~m},
$$

where we put $n=1$. Though the electron orbit (11) deeply penetrates into the middle of the proton, the electron still does not reach the critical distance of $2 \times 10^{-14} \mathrm{~m}$ that characterizes the quark orbit inside the proton [45]. If we put $n=2,3$, the radius (11) will be larger but still in the order of femtometers.

The system under consideration represents the rigid rotor problem, which is well known in quantum mechanics (see, e.g. Ref. 46). The Lagrangian of the rigid rotor reads

$$
L=\frac{1}{2}\left(m_{\mathrm{p}}+m_{\mathrm{e}}+m_{\mathrm{w}}\right) \bar{\rho}^{2}+\frac{1}{2} m \overline{\mathrm{p}}_{\mathrm{pe}}^{2}-U\left(r_{\mathrm{pe}}\right)
$$

where $\rho$ is the coordinate of the center-of-mass, $r_{\mathrm{p}-\mathrm{e}}$ is the relative coordinate between the proton and heavy electron (11), $\boldsymbol{m}$ is the reduced mass that is approximately equal to the proton mass $m_{\mathrm{p}}$, and $U\left(r_{\mathrm{p}-\mathrm{e}}\right)$ is the Coulomb potential. In the reference frame associated with the center-of-mass, coordinates of the proton and the electron are expressed via the relative coordinate $r_{\mathrm{p}-\mathrm{e}}$

$$
x_{\mathrm{e}}=\frac{m_{\mathrm{p}} x_{\mathrm{pe}}}{m_{\mathrm{p}}+m_{\mathrm{e}}}, \quad x_{\mathrm{p}}=-\frac{m_{\mathrm{e}} x_{\mathrm{pe}}}{m_{\mathrm{p}}+m_{\mathrm{e}}} .
$$

The moment of momentum of this two-particle system is $J=m_{\mathrm{p}} r_{\mathrm{pe}}^{2}$. Possible frequencies of radiation of the rigid rotator of \{proton + heavy electron $\}$ are

$$
\omega_{l, l-1}=\frac{\hbar}{m_{\mathrm{p}} r_{\mathrm{pe}}^{2}} l
$$

where $h /\left(m_{\mathrm{p}} r_{\mathrm{pe}}^{2}\right) \cong 4.78 \times 10^{20} \mathrm{~s}^{-1}$ and $l=1,2,3, \ldots$

The velocity of these \{proton + heavy electron\} pairs is thermal, which follows from the conditions at which they were created in the experiments of Don Borgi [23] and Santilli [24]. Namely, the pair was created with zero kinetic energy.

As has been discussed in Subsection 3.2, in our experiments the gamma-ray radiation has appeared as a result of a peculiar mechanism of scattering of protons by tungsten atoms of the cathode. On the other hand, if a tungsten atom collides with an incoming proton being in the antiphase, i.e. hits on the counter movement, we arrive at the situation that just has been considered above for Santilli's [24] experiment. Namely, if at the collision the equality holds, the proton will itself absorb the tungsten atom's inerton cloud and catching an electron it will form a \{proton + heavy electron \} pair. Let us consider the left and right hand sides of the relationship (15).

$$
m_{\mathrm{w}} v_{\mathrm{w}}=m_{\mathrm{p}} v_{\mathrm{p}}
$$

The thermal velocity of the tungsten atom at a room temperature is $v_{\mathrm{w}}=\sqrt{3 k_{\mathrm{B}} T / m_{\mathrm{w}}} \cong 2.018 \times 10^{2} \mathrm{~m} \cdot \mathrm{s}^{-1}$ and hence the momentum of the tungsten atom is $m_{\mathrm{W}} v_{\mathrm{W}}=$ $6.155 \times 10^{-23} \mathrm{~kg} \cdot \mathrm{m} \cdot \mathrm{s}^{-1}$.

The velocity of the incoming proton can be calculated by the expression $v_{\mathrm{p}}=\sqrt{2 e V / m_{\mathrm{p}}}$ where the voltage, which corresponds to the resonance interaction, is $17 \mathrm{~V}$. However, in the experiment we have dealt with a pulsing current, which due to the modulation should generate electric impedance at the edge surface of the cathode. Impedance slows the transfer of charge across the border gas plasma electrode, which in terms of capacitance reflects the capacity of the electrical double layer on the electrode. Slowing the motion of charges can be expressed as a tangent of the angle between the active and reactive resistance, $\tan \varphi$. Then the velocity of the proton becomes $v_{\mathrm{p}}=\sqrt{2 e V \tan \varphi / m_{\mathrm{p}}}$. A fitting value $\tan \varphi=\mathbf{0 . 4 1 6}$ makes it possible to compute the proton velocity $v_{\mathrm{p}}=3.68 \times 10^{4} \mathrm{~m} \cdot \mathrm{s}^{-1}$ that leads to the momentum $m_{\mathrm{p}} v_{\mathrm{p}}=6.155 \times 10^{-23} \mathrm{~kg} \cdot \mathrm{m} \cdot \mathrm{s}^{-1}$, which is equal to $m_{\mathrm{w}} v_{\mathrm{w}}$.

Thus, the relationship (15) indeed holds, which means that we in fact measured fluxes of \{proton + heavy electron pairs. Such \{proton + heavy electron \} pair can be named a subhydrogen, an additional term for the known hydrex [11-15], hydrino [16-19] and pseudoneutron [24]. Nevertheless, it seems the term subhydrogen is most appropriate as it indicates the subatomic size and properties of the \{proton + heavy electron $\}$ pair. The conditions at which our subhydrogens were created point out that they moved with low velocities.

In the experiment carried out with a helium gas, we observed practically the same phenomena: gamma quanta and a neutron flux. In this case instead of protons we dealt with $\boldsymbol{\alpha}$ particles. At the momentum resonance the expression (15) looks as follows: $m_{\mathrm{w}} v_{\mathrm{w}} \cong 4 m_{\mathrm{p}} v_{\alpha}$, because the mass of $\alpha$ particle is $\boldsymbol{m}_{\boldsymbol{\alpha}} \cong \mathbf{4} \boldsymbol{m}_{\mathrm{p}}$. The resonance was reached at $V \approx 15 \mathrm{~V}$, which means that in the expression for velocity $v_{\alpha}=\sqrt{2 \cdot(2 e) \cdot V \cdot \tan \varphi_{\alpha} /\left(4 m_{\mathrm{p}}\right)}$ of the $\alpha$ particle we have to put $\tan \varphi_{\alpha}=\mathbf{0 . 1 5 7}$. Hence the resonance conditions have resulted into the creation of $\left\{{ }_{2}^{4} \mathrm{He}^{2+}+\right.$ two heavy electrons pairs. Such kind of a subatomic particle can be named a subhelium.

Since the unit for detection of neutron radiation BDPN-07 measured synthesized neutral subatomic particles subhydrogens in a hydrogen gas and subheliums in a helium gas - we have to try to understand what kinds of nuclear reactions may occur in the unit BDPN-07.

Basically, Helium-3 proportional counters utilize the following reaction for the detection of thermal neutrons: 
${ }^{3} \mathrm{He}+n^{0}$ (thermal) $\rightarrow{ }^{1} \mathrm{H}+{ }^{3} \mathrm{H}+\mathrm{Q},(16)$

where $\mathbf{Q}$ is a quantum of energy (typically this is a gamma quantum, which for the reaction above has the energy 764 $\mathrm{keV})$.

In the case when instead of a neutron we have a subhydrogen, the reaction (16) may look like this

${ }^{3} \mathrm{He}+\langle n\rangle_{\mathrm{H}}$ (thermal) $\rightarrow{ }^{1} \mathrm{H}+\left\{{ }^{2} \mathrm{H},\langle n\rangle_{\mathrm{H}}\right\}+\mathrm{Q},(17)$

where $\langle n\rangle_{\mathrm{H}} \equiv\left\{p^{+}\right.$, heavy $\left.e^{-}\right\}$is the subhydrogen and $\left\{{ }^{2} \mathrm{H},\langle n\rangle_{\mathrm{H}}\right\}$ is the neutral subatomic particle formed by the deuteron and subhydrogen, i.e. the \{deuteron + subhydrogen pair.

In the case when instead of a neutron we have a subhelium, the reaction (16) may look as follows

${ }^{3} \mathrm{He}+\langle n\rangle_{\text {Hе }}$ (thermal) $\rightarrow{ }^{1} \mathrm{H}+\left\{{ }^{2} \mathrm{H},\langle n\rangle_{\text {не }}\right\}+\mathrm{Q},($

where $\langle n\rangle_{\mathrm{He}} \equiv\left\{{ }_{2}^{4} \mathrm{He}^{2+}\right.$, two heavy $\left.e^{-}\right\}$is the subhelium and $\left\{{ }^{2} \mathrm{H},\langle n\rangle_{\mathrm{He}}\right\}$ is the neutral \{deuteron + subhelium\} pair.

The nuclear pairs $\left\{{ }^{2} \mathrm{H},\langle n\rangle_{\mathrm{H}}\right\}$ and $\left\{{ }^{2} \mathrm{H},\langle n\rangle_{\mathrm{He}}\right\}$ may imitate a real tritium ${ }^{3} \mathbf{H}$

The reduced mass in the present subhelium is about $\mathbf{4} m_{\mathrm{p}}$, which permits one to estimate the size of subhelium by using the expression for the Bohr radius (11). If we put the number of orbit $n=2$, we will get exactly the same radius, as is the case for the subhydrogen (11).

In Mills' experiments [16-19] the relationship (15) also comes true. In this case we put for the proton $v_{\mathbf{p}}=\sqrt{2 e V / m_{\mathrm{p}}}$ and at $V=1.5 \mathrm{~V}$ the proton momentum equals $m_{\mathrm{p}} v_{\mathrm{p}}=1.306 \times 10^{-23} \mathrm{~kg} \cdot \mathrm{m} \cdot \mathrm{s}^{-1}$. For the tungsten atom, which diffuses in a hydrogen gas, one should include in the expression for the velocity a factor $C$ related to the diffusion coefficient; namely, we have to rewrite the velocity of the tungsten atom as follows: $v_{\mathrm{w}}=\sqrt{3 k_{\mathrm{B}} T C / m_{\mathrm{w}}}$. Putting $C=0.212$, we calculate for the tungsten atom the same momentum $m_{\mathrm{w}} v_{\mathrm{w}}=1.306 \times 10^{-23} \mathrm{~kg} \cdot \mathrm{m} \cdot \mathrm{s}^{-1}$.

In the experiments of Taleyarkhan, Nigmatulinet et al. [2-8] neutrons and tritium atoms emitted from the collapsed bubble in a quantity of $150-200$ [8] for each kind of species per one pulse. This is also in line with the model described above. As follows from expression (8), each of $N \approx 3 \times 10^{2}$ heavy inertons transfers the energy $\varepsilon=1.5 \times 10^{-13} \mathrm{~J}$ to gas entities in the collapsed bubble. Some of those entities, namely, molecules of acetone/benzene absorb the inertons and radiate gamma quanta as has been discussed in Subsection 3.2. The other entities, rather molecules/atoms of deuterium, could emerge in the bubble owing to the destruction of deuterated acetone/benzene molecules by an outside neutron flux or $\boldsymbol{\alpha}$ particles from the uranium salt. It seems reasonable to assume that at the collapse, the inerton clouds are stripped from deuteron atoms, which are present in the $N \approx 3 \times 10^{2}$ molecules of the boundary layer that separates the liquid from the gas. Thus a deuterium, which has only one electron, absorbs a heavy inerton cloud and a pair of the \{deuteron + heavy electron \} is formed; below such pair is named the subdeuterium. The reduced mass of the subdeuterium is equal to almost $2 m_{\mathrm{p}}$. Then the size $r_{\mathrm{de}}$ of the subdeuterium may be twice the radius (11) at the orbit number $n=2$.

The reaction (16) in the case of a synthesized subdeuterium, which acts instead of a natural neutron, may look as follows

$$
{ }^{3} \mathrm{He}+\langle n\rangle_{\mathrm{D}} \text { (thermal) } \rightarrow{ }^{1} \mathrm{H}+\left\{{ }^{2} \mathrm{H},\langle n\rangle_{\mathrm{D}}\right\}+\mathrm{Q}
$$

where $\langle n\rangle_{\mathrm{D}} \equiv\left\{{ }^{2} \mathrm{H}^{+}\right.$, heavy $\left.e^{-}\right\}$is the subdeuterium and $\left\{{ }^{2} \mathrm{H},\langle n\rangle_{\mathrm{D}}\right\}$ is the $\{$ deuteron + subdeuterium $\}$ pair.

The kinetic energy of the heavy inerton cloud $\varepsilon=4 \cdot 10^{-13} \mathrm{~J}$ continues to push the whole pair. Indeed, if the inerton cloud was stripped from a deuteron atom, we may write the kinetic energy of the cloud in the form of $2 m_{\mathrm{p}} v_{\text {inet }}^{2} / 2=4 \cdot 10^{-13} \mathrm{~J}$, which defines the velocity $v_{\text {inert }}=1.55 \times 10^{7} \mathrm{~m} \mathrm{~s}^{-1}$. In the subdeuterium the total mass is $2 m_{\mathrm{p}}+\left(2 m_{\mathrm{p}}+m_{e}\right) \simeq 4 m_{\mathrm{p}}$ and its center-of-mass can continue to move with the same kinetic energy equal to $4 \times 10^{-13} \mathrm{~J}$, which is $2.5 \mathrm{MeV}$ (as was revealed in the experiments of Taleyarkhan, Nigmatulin et al. [2-8]), though with a little bit lower velocity $10^{7} \mathrm{~m} \mathrm{~s}^{-1}$.

The known nuclear reaction

$$
p^{+}+e^{-} \rightarrow n^{0}+v_{e}
$$

cannot really be applied for the subhydrogen, as the reaction (20) occurs only in a large nucleus when the nucleus catches an orbital electron from the $\mathrm{K}$ or $\mathrm{L}$ shell, which results in the decay of an inner proton to a neutron. In the subhydrogen it is the huge mass of the electron, which protects the proton from the direct Coulomb invasion of the electron (indeed from expressions (11) and (14) we derive the inequality $\left.\hbar \omega_{l, l-1} \geq e^{2} /\left(4 \pi \varepsilon_{0} r_{\mathrm{pe}}\right)\right)$.

Regarding tritium detected in works [2-8], we may assume the usual reaction

$$
{ }_{1}^{2} \mathrm{D}+{ }_{1}^{2} \mathrm{D} \rightarrow{ }_{1}^{2} \mathrm{~T}(1.01 \mathrm{MeV})+p^{+}(3.02 \mathrm{MeV})
$$

or maybe the nuclear pair $\left\{{ }^{2} \mathrm{H},\langle n\rangle_{\mathrm{D}}\right\}$ determined above, which is formed of the deuteron and subdeuterium pair, imitating tritium ${ }_{1}^{2} \mathrm{~T}$.

One more interesting reaction at sonofusion was reported by FitzGerald [47]; experimenting with sonoluminescence in a liquid $\mathrm{D}_{2} \mathrm{O}$, he measured an output of $\boldsymbol{\alpha}$ particles, i.e. the low energy nuclear reaction ${ }_{1}^{2} \mathrm{D}+{ }_{1}^{2} \mathrm{D}={ }_{2}^{4} \mathrm{He}$ took place. Or maybe that was an imitation of the reaction. In any case it seems the FitzGerald's result has not been replicated by other researchers yet.

\subsection{Neutrons generated by piezocrystals}

Putterman's group [48] reported that a pyroelectric crystal $\mathrm{LiTaO}_{3}$ heated to $25^{\circ} \mathrm{C}$ in a low-pressure deuterated 
atmosphere $\left(\begin{array}{lll}0.7 & \mathrm{~Pa}\end{array}\right)$ generated fusion under desktop conditions. The electrostatic field of the crystal generated and accelerated a deuteron beam $(>100 \mathrm{keV}$ and $>4 \mathrm{nA})$. The beam was focused by a tungsten needle. The ionized deuterium was accelerated into a target of erbium deuteride $\left(\mathrm{ErD}_{2}\right)$ and upon striking the target produced a neutron flux that was over 400 times the background level. The presence of neutrons from the reaction was confirmed by pulse shape analysis and proton recoil spectroscopy.

$$
{ }_{1}^{2} \mathrm{D}+{ }_{1}^{2} \mathrm{D} \rightarrow{ }_{2}^{3} \mathrm{He}(820 \mathrm{keV})+n^{0}(2.45 \mathrm{MeV})
$$

Geuther et al. [49] obtained results similar to Putterman's [48] by experimenting with pyroelectric crystals $\mathrm{LiNbO}_{3}$ and $\mathrm{LiTaO}_{3}$; in particular $2.5 \mathrm{MeV}$ neutrons were detected. Danon [50] by using a similar device was able to generate $\mathrm{X}$-rays with energy over $200 \mathrm{keV}$ and about $10^{5}$ neutrons per heating cycle. Besides, pyroelectric crystal accelerators were shown to be a viable way of producing D-D and D-T neutron, up to $10^{7}$ neutrons per second.

At last Putterman's group [51] extended the earlier work with deuterated targets and reported a $14.1 \mathrm{MeV}$ neutron signal far above background.

Cardone et al. [52] informed that measured emission of neutrons at a mechanical crushing of solid specimens, such as marble and granite; neutron emission measurements were conducted by means of helium-3 neutron detectors. Besides, Cardone et al. [53] reported the results of neutron measurements carried out during the application of ultrasounds to a solution containing only stable elements like Iron and Chlorine, without any other radioactive source of any kind; they called the phenomenon "piezonuclear neutrons". Piezonuclear decay of thorium was measured by Cardone and Mignani [54, 55] at cavitation of a solution of thorium-228 in water; the transformation occurred at a rate $10^{4}$ times faster than the natural radioactive decay would do.

Note that, operating with the crystal $\mathrm{LiNbO}_{3}$, namely, illuminating its surface with a laser beam, we [38] generated photoelectrons that assembled in a cluster. About $10^{10}$ electrons held in the cluster by means of inertons that also were knocked out of the crystal studied by the same laser beam. An emission of X-ray was observed as well. In the experiment we demonstrated the appearance of a rather powerful inerton field emanating from the crystal. It was that bunch of inertons, which bonded electrons in the cluster.

Therefore in experiments [48-55] inerton fields should also be generated when bombarding the target by deuterons. Inertons appeared also at the striction of the piezocrystals (because the rate of striction is much faster than the sound velocity, see also Ref. 56). Thus the interaction of deuterons with generated inertons occurred in accordance with the scheme described above in Section 3.2. The generation of X-rays was caused by the absorption of inertons by the atoms' electrons with the subsequent emission of X-rays, as is the case with the emission of gamma-quanta described in Section 3.1 and the ultraviolet photons, as was examined [1] at the consideration of sonoluminescence. By this means the observed neutrons [48-55] were not real neutrons but subatoms and nuclear pairs, which follows from the discussion in the previous subsections of the present work.

\section{Concluding remarks}

Thus we in fact have generated and measured a mysterious particle, which was previously revealed by many researchers dealing with plasma, cold fusion transformations in condensed media, sonoluminescence and discharges generated by piezocrystals. Inexplicable particles were generated in our experiments in a hydrogen gas, helium gas and the air. Each of these subatomic particles is formed on the basis of a proton, deuteron or $\boldsymbol{\alpha}$ particle and an electron (or a pairs of electrons as is the case for $\boldsymbol{\alpha}$ particle) in the presence of an inerton cloud released from one of the nearest atoms. It seems the best general name for such particles is a subatom, such as subhydrogen, subdeuterium and subhelium. Moreover, these particles are able to mate with a proton, neutron and deuteron, forming nuclear pairs whose behavior can be described within the rigid rotor problem.

Can similar more or less stable subatoms and nuclear pairs be obtained on the basis of an atom with at least a few electrons? The question is open. To answer the question, one has to examine discharges in different gases in an experiment similar to ours, which has been described in the present paper.

The physics of subatoms and nuclear pairs is very new and derived from a submicroscopic concept of the real physical space and submicroscopic mechanics, which for years have been developed by one of the authors (V. K.). The submicroscopic concept associates the nuclear forces with both direct coalescence of surfaces of the nucleons and the overlapping of inerton clouds of the nucleons at their motion through the space tessellattice [57, 45]. Inerton fields can be generated artificially, which could result in some nuclear reactions [45]. A large mass concentrated in a subatom owing to adsorbed inertons is able to affect nuclear reactions overcoming the Coulomb barrier, in particular, the reactions of fission and fusion. In any case subatoms and nuclear pairs formed with the participation of subatoms imitate nuclear reactions in a way similar to that of usual neutrons [12-15,9]. We may assume that subatoms and nuclear pairs may stimulate nuclear transmutations. In particular a number of spontaneous transmutations were observed in biological systems [58] and also in iron irradiated by an intensive inerton field [45].

The results obtained point to the fact that nuclear physics and particle physics must more tightly cooperate with condensed matter physics both in physics of nuclear fission and fusion.

The study for fusion energy in the framework of the pure thermonuclear concept, when fusion reactions are taking place in plasma, becomes more and more problematic. Such reactions require control, which is rather impossible to do in rarefied plasma. In dense plasma the inerton field should play an important role. However, it has so far been out of 
consideration by physicists. Besides, it should be emphasized that in nature we do not observe sustained fusion reactions in a pure plasma state; stars and in particular the Sun consist rather of a metallic liquid than gaseous plasma [59].

In the case of low energy nuclear reactions we also meet a number of problems and one of them is associated with non-radiating transformations of particles. The majority of such reactions do not generate heat and they cannot be considered as a prelude for future industrial power plants. Nevertheless, we have to mention a successful attempt of Rossi [60]. The present paper allows one to go ahead planning experiments with predetermined parameters (for example, such as a tenfold increase in the energy output, etc.).

\section{Acknowledgments}

The authors express their gratitude to Indra Scientific SA, a company based in Brussels, which initiated and financially supported the present study.

\section{REFERENCES}

[1] V. Krasnoholovets, Sound into light: on the mechanism of the phenomenon of sonoluminescence, J. Advan. Phys. 5, No. 2, 168-175 (2016).

[2] R. P. Taleyarkhan, C. D. West, J. S. Cho, R. T. Lahey (Jr.), R. I. Nigmatulin and R. C. Block, Evidence for nuclear emissions during acoustic cavitation. Science 295, 1868-1873 (2002).

[3] R. P. Taleyarkhan, C. D. West, R. T. Lahey (Jr.), R. I. Nigmatulin, R. C. Block and Y. Xu, Nuclear emissions during self-nucleated acoustic cavitation, Phys. Rev. Lett. 96, 034301(4) (2006).

[4] R. P. Taleyarkhan, R. C. Block, R.T. Lahey (Jr.), R. I. Nigmatulin and Y. Xu, Reply to [Lipson] 'Comment on "Nuclear Emissions During Self- Nucleated Acoustic Cavitation"'. Phys. Rev. Lett. 97, 149402 (2006)

[5] R. P. Taleyarkhana, J. Lapinskasa, Y. Xua, J. S. Chob, R. C. Blockc, R. T. Lahey (Jr.) and R. I. Nigmatulind, Modeling, analysis and prediction of neutron emission spectra from acoustic cavitation bubble fusion experiments, Nuclear Engineering and Design 238, 2779-2791 (2008).

[6] R. P. Taleyarkhan, C. D. West, R. T. Lahey (Jr.), R. I. Nigmatulin, R. C. Block, J. S. Cho and Y. Xu, Recent advances and results in acoustic inertial confinement bubble nuclear fusion. In: Marwan, J., Krivit, S.B. (eds.) American Chemical Society Symposium Series: Low-Energy Nuclear Reactions and New Energy Technologies Sourcebook (Vol. 2), pp. 139-157. American Chemical Society/Oxford University Press, Washington, D.C. (2009).

[7] R. P. Taleyarkhan, C. D. West, J. Cho, R. T. Lahey (Jr.), R. I. Nigmatulin and R. C. Block, Comments on Letter (Phys. Rev. L, Vol. 89, No. 10, 2002) by D. Shapira \& M. Saltmarsh,
arXiv:1307.3217 [physics.gen-ph] (2013).

[8] R. I. Nigmatulin, R. T. Lahey (Jr.), R. C. Block, R. P. Taleyarkhan and C.D. West, On thermonuclear processes in cavitation bubbles, Uspekhi Fizich. Nauk 184, 947-960 (2014); in Muscovian.

[9] E. Storms, The science of low energy nuclear reaction. A comprehensive compilation of evidence and explanations about cold fusion, World Scientific Publishing, Singapore (2007).

[10] D. J. Nagel, Lattice-enabled nuclear reactions in the nickeland hydrogen gas system, Current Science 108, No. 4, 646-652 (2015).

[11] J. Dufour, Cold fusion by sparking in hydrogen isotopes, Fusion Technol. 24, 205-228 (1993).

[12] J. Dufour, J. Foos, J. P. Millot, X. Dufour, Interaction of palladium/hydrogen and palladium/deuterium to measure the excess energy per atom for each isotope, Fusion Technol. 31, 198-209 (1997).

[13] J. Dufour, D. Murat, X. Dufour and J. Foos, Hydrogen triggered exothermal reaction in uranium metal, Phys. Lett. A 270, 254-264 (2000).

[14] J. Dufour, D. Murat, X. Dufour and J. Foos, Exothermic reaction induced by high density current in metals - Possible nuclear origin, Ann. Fond. L. de Broglie 29, 1081-1093 (2004).

[15] J. J. Dufour, X. J. C. Dufour and J. D. Vinko, Pico-chemistry: The possibility of new phases in some hydrogen/metal systems. Int. J. Mod. Phys. B 27, 1362038[8 pages] (2013).

[16] R. Mills, J. Dong and Y. Lu, Observation of extreme ultraviolet hydrogen emission from incandescently heated hydrogen gas with certain catalysts, Int. J. Hydrogen Energy $25,919-943(2000)$

[17] R. Mills, B. Dhandapani, M. Nansteel, J. He, A. Voigt, Identification of compounds containing novel hydride ions by nuclear magnetic resonance spectroscopy, Int. J. Hydrogen Energy 26, 965-979 (2001).

[18] R. Mills and M. Nansteel, P. Ray, Argon-hydrogen-strontium discharge light source, IEEE Transact. Plasma Sci. 30, no. 2 , 639-653 (2002).

[19] R. L. Mills, P. Ray, B. Dhandapani, R. M. Mayo and J. He, Comparison of excessive Balmer alpha line broadening of glow discharge and microwave hydrogen plasmas with certain catalysts, J. Appl. Phys. 92, 7008-7022 (2002).

[20] H. Conrads, R. Mills and Th. Wrubel, Emission in the deep vacuum ultraviolet from a plasma formed by incandescently heating hydrogen gas with trace amounts of potassium carbonate, Plasma Sources Science and Technology 12, 389-395 (2003).

[21] R. Mills, M. Nansteel and P. Ray, Excessively bright hydrogen-strontium plasma light source due to energy resonance of strontium with hydrogen, J. of Plasma Physics 69, 131-158 (2003).

[22] R. Mills, P. Ray, B. Dhandapani, W. Good, P. Jansson, M. Nansteel, J. He and A. Voigt, Spectroscopic and NMR identification of novel hydride ions in fractional quantum energy states formed by an exothermic reaction of atomic hydrogen with certain catalysts, Eur. Phys. J. -App. Phys. 28, 


\section{3-104 (2004).}

[23] C. Borghi, C. Giori, A. A. Dall'Ollio, Experimental evidence of emission of neutrons from cold hydrogen plasma, American Institute of Physics (Phys. At. Nucl.) 56, no 7 (1993).

[24] R. M. Santilli, Confirmation of Don Borghi's experiment on the synthesis of neutrons from protons and electrons, Preprint IBR-EP-39 of 12-25-06; arXiv.org: physics/0608229 (2006).

[25] E. Conte, M. Pieralice, An experiment indicates the nuclear fusion of the proton and electron into a neutron, Infinite Energy 4, 67 (1999).

[26] L. I. Urutskoev, V. I. Liksonov, and V. G. Tsinoev, Experimental discovery of a "strange" radiation and the transformation of chemical elements, Zhurnal Radioelektroniki, no. 3 (2000), http://jre.cplire.ru/iso/mar00/4/text.html; in Muscovian

[27] L. I. Urutskoev, V. I. Liksonov and V. G. Tsinoev, Observation of transformation of chemical elements during electric discharge, Ann. Fond. L. de Broglie 27, 701-726 (2002).

[28] V. Krasnoholovets, Submicroscopic viewpoint on gravitation, cosmology, dark energy and dark matter, and the first data of inerton astronomy, in Recent Developments in Dark Matter Research. N. Kinjo and A. Nakajima (eds.), pp. 1-61, Nova Science Publishers, N. Y. (2014).

[29] M. Bounias and V. Krasnoholovets, Scanning the structure of ill-known spaces: Part 1. Founding principles about mathematical constitution of space, Kybernetes: The Int. J. Systems \& Cybernetics 32, no. 7/8, 945-75 (2003), L. Feng, B. P. Gibson and Yi Lin (eds.); arXiv:physics/0211096.

[30] M. Bounias and V. Krasnoholovets, Scanning the structure of ill-known spaces: Part 2. Principles of construction of physical space, ibid. 32, no. 7/8, 976-1004 (2003), L. Feng, B. P. Gibson and Yi Lin (eds.); arXiv:physics/0212004.

[31] M. Bounias and V. Krasnoholovets, Scanning the structure of ill-known spaces: Part 3. Distribution of topological structures at elementary and cosmic scales, ibid. 32, no. 7/8, 1005-20 (2003), L. Feng, B. P. Gibson and Yi Lin (eds.); arXiv:physics/0301049.

[32] M. Bounias and V. Krasnoholovets, The universe from nothing: A mathematical lattice of empty sets. Int. J. Anticipatory Computing Systems, D. Dubois (ed.) 16, 3-24 (2004); arXiv:physics/0309102.

[33] V. Krasnoholovets and D. Ivanovsky, Motion of a particle and the vacuum, Phys. Essays 6, 554-563 (1993); arXiv.org:quant-ph/9910023.

[34] V. Krasnoholovets, Motion of a relativistic particle and the vacuum, Phys. Essays 10, 407-416 (1997); arXiv.org:quant-ph/9903077.

[35] V. Krasnoholovets, Submicroscopic deterministic quantum mechanics, Int. J. Comput. Anticipat. Systems, D. Dubois (ed.) 11, 164-179 (2002); arXiv:quant-ph/0109012.

[36] V. Krasnoholovets, S. Skliarenko and O. Strokach, On the behavior of physical parameters of aqueous solutions affected by the inerton field of Teslar ${ }^{\circledR}$ Technology, Int. J. Mod. Phys. B 20, No. 1, 1-14 (2006); arXiv:0810.2005.

[37] E. Andreev, G. Dovbeshko and V. Krasnoholovets, The study of influence of the Teslar technology on aqueous solution of some biomolecules, Research Lett. Phys. Chem., Vol. 2007, Article ID 94286, 5 pages; arXiv:1204.6062 [physics.gen-ph].

[38] V. Krasnoholovets, N. Kukhtarev and T. Kukhtareva, Heavy electrons: Electron droplets generated by photogalvanic and pyroelectric effects, Int. J. Mod. Phys. B 20, No. 16, 2323-2337 (2006); arXiv:0911.2361V.

[39] Krasnoholovets, Inerton field effects in nanosystems, in Quantum Nanosystems: Structure, Properties and Interactions, M. V. Putz (ed.), pp. 59-102. Apple Academic Press, Toronto (2014).

[40] V. Krasnoholovets, Sub microscopic description of the diffraction phenomenon, Nonlin. Opt. Quant. Opt. 41, pp. 273-286 (2010); arXiv:1407.3224 [physics.gen-ph].

[41] V. Krasnoholovets, Variation in mass of entities in condensed media, Appl. Phys. Research 2, 46-59 (2010), http://www.ccsenet.org/journal/index.php/apr/article/view/42 87.

[42] M. Davidson, Theories of variable mass particles and low energy nuclear phenomena, Found. Phys. 44, 144-174 (2014).

[43] M. Davidson, Variable mass theories in relativistic quantum mechanics as an explanation for anomalous low energy nuclear phenomena, J. Physics: Conference Series 615, 012016 (2015).

[44] V. Krasnoholovets, On the nature of the electric charge, Hadronic J. Suppl., 18, No. 4, 425-456 (2003); arXiv:physics/0501132.

[45] V. Krasnoholovets, Quarks and hadrons in the real space, J. Advan. Phys. 5 (2016), in press.

[46] A. A. Sokolov, Yu. M. Loskutov and I. M. Ternov, Quantum Mechanics, 2d edition, Prosveshchenie, Moscow, 1965, p. 222 (in Muscovian).

[47] F. B. FitzGerald, Sonoluminescence and sonofusion - how and why. The General Science J., 16 Jan 2009; http://www.gsjournal.net/Science-Journals/Essays/View/192 2 .

[48] B. Naranjo, J. K. Gimzewski and S. Putterman, Observation of nuclear fusion driven by a pyroelectric crystal, Nature 434, 1115-1117 (2005)

[49] J. Geuther, Y. Danon and F. Saglime. Nuclear reactions induced by a pyroelectric accelerator. Phys. Rev. Lett. 96, 054803 (2006).

[50] Y. Danon, Pyroelectric crystal D-D and D-T neutron generators. J. Instrumentation (JINST) 7, C04002 [4 pages] (2012).

[51] B. Naranjo, S. Putterman and T. Venhaus, Pyroelectric fusion using a tritiated target. Nuclear Instruments and Methods in Physics Research Section A: Accelerators, Spectrometers, Detectors and Associated Equipment 632, $43-46$ (2011).

[52] F. Cardone, A. Carpinteric, and G. Lacidogna, Piezonuclear neutrons from fracturing of inert solids, Phys. Lett. A 373, 4158-4163 (2009).

[53] F. Cardone, G. Cherubini and A. Petrucci, Piezonuclear neutrons, Phys. Lett. A 373, 862-866 (2009). 
[54] F. Cardone, R. Mignani and A. Petrucci, Piezonuclear decay of thorium, Phys. Lett. A 373, 1956-1958 (2009).

[55] F. Cardone, R. Mignani and A. Petrucci, Reply to "Comment on 'Piezonuclear decay of thorium' [Phys. Lett. A 373 (2009) 1956]" [Phys. Lett. A 373 (2009) 3795], Phys. Lett. A 373, 3797-3800 (2009).

[56] V. Krasnoholovets and I. Gandzha, A submicroscopic description of the formation of crop circles, Chaotic Modeling and Simulation (CMSIM) 2, 323-335 (2012), http://www.cmsim.eu/April_2012_issue.html.

[57] V. Krasnoholovets, Reasons for nuclear forces in light of the constitution of the real space. Scientific Inquiry 7, 25-50
(2006); arXiv:1104.2484 [physics.gen-ph]

[58] C. L. Kervran, Biological Transmutations, second printing, J. de Langre (ed.). Happiness Press, Magalia, California (1998).

[59] P.-M. Robitaille, Forty lines of evidence for condensed matter - the Sun on trial: liquid metallic hydrogen as a solar building block, Progress in Physics (2013) Vol. 4, 90-142.

[60] A. Rossi, International Patent Application WO 2009125444 , Method and Apparatus for carrying out nickel and hydrogen exothermal reactions (2009). 